\title{
ACCENT D'INTENSITÉ ET ACTION INTONATIVE EN FRANÇAIS MODERNE
}

Cet article se donne pour objectif d'exposer une réflexion critique sur la nature, le fonctionnement et les interactions possibles de deux faits prosodiques en français: l'accent d'intensité et l'intonation de la phrase. Les effets de l'accentuation par intensité se rangent au niveau syntagmatique de la chaîne parlée (donc au niveau du mot phonétique). Le fonctionnement de l'intonation, en français, affecte globalement la phrase toute entière, mais les composantes élémentaires de ses formes globales touchent à la structuration syntaxique, formant ainsi des unités intonatives qui «couvrent» les mêmes éléments que l'accent linguistique. Il s'agit donc d'examiner les rapports qu'il pourrait y avoir entre la réalisation des formes intonatives et les fonctions de l'accent linguistique, étant donné que les deux faits prosodiques, à l'intérieur de la chaîne parlée française, se partagent un même champ d'opération.

\section{Nature et fonctions de l'accent dit «accent d'intensité» en français}

Selon l'acception générale et traditionnelle, l'accent fonctionnel linguistique du français est désigné sous le nom d' «accent d'intensité». ${ }^{1}$ Le terme tend à mettre en évidence le rôle crucial que l'un des trois paramètres prosodiques principaux, à savoir l'énergie (intensité) de la voix émise, joue à la réalisation de ce phénomène. Du point de vue substanciel (physique), l'accent d'intensité serait donc la mise en valeur d'une et d'une seule syllabe par rapport aux autres constituants syllabiques d'une chaîne parlée déterminée, cette mise en valeur étant effectuée au moyen d'une intensité vocalique ou force expiratoire accrue. En français, l'accent linguistique est à juste titre considéré comme un fait prosodique relevant du niveau du syntagme. Il est généralement avancé qu'en français, l'accent d'intensité affecte irrévocablement et fixement la dernière syllabe de l'unité accentuelle, ${ }^{2}$ donc du syntagme. Les phonéticiens ont également

I Dans une terminologie «classique», l'accent d'intensité est également connu sous d'autres dénominations, notamment comme accent d'énergie, accent expiratoire, accent dynamique, accent de force ou, par glissement du sens original, accent tonique. Toutes ces désignations soutiennent que ce type d'accent est réalisé au moyen d'une force expiratoire relativement augmentée.

2 L'unité accentuelle (quels que soient la nature et le rôle de l'accent dans une langue donnée) est considérée ici comme un élément plus ou moins long d'une chaîne parlée, affecté d'une seule syllabe fonctionnellement accentuée, et réalisé normalement en une seule émission de l'air expiré. 
l'habitude d'accorder à la syllabe accentuée une forte tendance vers l'allongement, c'est-à-dire vers une augmentation physique de la longueur qui résulte en une durée proéminente de la syllabe affectée d'accent par rapport aux syllabes dites inaccentuées.

En termes prosodiques, visant à rendre compte de la fonction de l'accent linguistique en français, donc dudit «accent d'intensité», le phénomène discuté exerce d'abord le rôle de mise en relief de la syllabe accentuée, rôle relatif à sa valeur culminative. Cette fonction a pour but de distinguer la réalisation d'un sommet physique de l'unité accentuelle par rapport à son reste «inaccentué».

La seconde fonction que devrait remplir l'accent linguistique s'effectuerait par l'activation de sa valeur démarcative ou délimitative: en français, l'accent d'énergie est censé indiquer la fin du mot phonétique, puisqu'il paraît frapper régulièrement sa dernière syllabe. Ici, on essaiera cependant de montrer quelles sont, dans la réalisation de la chaîne parlée, les conséquences réelles de cette fameuse fixité de l'accent d'intensité français. ${ }^{3}$ Il paraît justifié d'avancer que la fixité absolue de n'importe quel fait linguistique (et plus encore, prosodique) détermine la réalisation de ce même fait jusqu'au point où elle devient généralement prédictible.

L'examen linguistique général du fonctionnement de $1^{\text {'accentuation }}{ }^{4}$ attribue à l'accent d'énergie une troisième fonction, et notamment celle qui découle de sa valeur distinctive. Or il est important de préciser tout de suite que la distinctivité présumée de l'accent ne saurait être réellement associée à la valeur distinctive qui, selon les principes de pertinence phonologique, est inhérente aux propriétés essentielles du phonème. Fonctionnant dans la chaîne parlée au niveau contrastif, l'accent d'énergie peut être distinctif dans les langues où il est également considéré comme mobile ou «non-fixe», comme en slovène, en anglais, en russe, ou, d'ailleurs, dans la plupart des langues romanes. Dans ces cas-là il peut, à la rigueur, former la base de construction de paires minimales contrastives (esp. 'termino, ter'mino, termi'no; ang. 'present, pre'sent; slov. 'gora, go'ra). Toujours est-il qu'il opère ses contrastes non pas sur le principe de substitution formelle, mais selon, pour ainsi dire, la règle «d'être ou ne pas être». La distinctivité ${ }^{5}$ de l'accent n'entrera donc pas en jeu quand on tentera de décrire le

3 Il semble utile de signaler en passant que ce genre d'idéalisations, souvent provenant d'une attitude non-critique propre à l'enseignement grammatical «latin», est adapté aux besoins didactiques qui sont à la base du transfert des connaissances linguistiques générales à une grande plupart des niveaux scolaires.

4 Évidemment, ce texte s'abstiendra clairement de traiter de diverses manifestations d'accent, réalisé au moyen de la variation tonale opérant, selon le principe de substitution, sur le développement d'une seule voyelle, c'est-à-dire de l'accent tonématique qui figure sous forme phonologique de tonème, propre aux systèmes prosodiques des langues à ton.

5 Dans cet article, la notion de distinctivité est utilisée à tour de rôle dans l'une de ses trois significations possibles. En termes phonologiques, la valeur distinctive est la qualité qu'a un phonème (ou l'un de ses traits) et sans laquelle un élément phonologique ne pourrait pas figurer dans le rôle d'objet de substitution ou de commutation sur l'axe paradigmatique de la chaîne parlée, entraînant ainsi un changement de sens. Dans l'acception accentologique, l'accent d'intensité n'est distinctif que dans les langues à accent mobile. Cette valeur est traitée de qualité par laquelle un 
fonctionnement de l'accent d'intensité en français, puisqu'il n'est guère en position, grâce aussi à sa fixité présumée, d'apporter une possibilité de distinction entre deux sens différents.

Comme il a déjà été indiqué, l'accent d'énergie n'a pas de statut phonologique proprement dit; il na donc pas la valeur de pertinence que pourrait lui attribuer la phonologie structurale linéaire. Celle-ci ne prend en considération que les manifestations phonétiques que l'on peut, conformément aux résultats qu'elles donnent dans une chaîne parlée, systémiser selon leur fonctionnement sur l'axe paradigmatique. Cela signifie qu'un phonème ou un de ses traits articulatoires ou acoustiques (audio-perceptifs) peut être substitué ou commuté par un autre élément du même ordre phonologique, recevant ainsi une valeur distinctive phonologique, et formant la base de construction de paires minimales (p. ex.: père - mer). La nature même de la manifestation accentuelle (hormis toujours les tonèmes) est telle qu'une syllabe déterminée (accentuable) porte ou ne porte pas l'accent d'intensité. Cela fait de ce type d'accent linguistique - qu'il soit fixe ou non-fixe - un phénomène prosodique qui exerce ses fonctions sur l'axe horizontal de la chaîne parlée et qui, par conséquent, n'a qu'une valeur contrastive. Le fonctionnement de l'accent d'énergie paraît particulièrement limité en français où la possibilité de mettre en contraste les syllabes est diminuée par la position immobile de l'accent. Il semble en plus que cette position fixe et absolument prévisible, position donc propre à la construction de l'unité accentuelle française, peut même avoir pour conséquence une certaine supposition de diminution de la fonction démarcative: ce type de positionnement de l'accent linguistique sur la dernière syllabe de l'unité correspond précisément à l'emplacement de la conclusion intonative. Autrement dit, l'accent affecte régulièrement et prévisiblement la syllabe sur laquelle se situe l'événement intonatif, déterminant la forme intonative qui se réalise sur une unité de souffle donnée.

\section{Quelques notions générales sur l'intonation de la phrase française ${ }^{6}$}

La ligne de déclinaison, la configuration du contour global et la conclusion d'une forme intonative représentent les traits principaux qui déterminent les formes prototypiques de l'intonation globale au niveau de la phrase. La ligne de déclinaison est une ligne abstraite qui relie les hauteurs tonales moyennes des voyelles initiales et finales dans le cadre d'une modalité et rend compte de la différence globale entre elles. C'est une ligne qui détermine en principe la modalité de l'énoncé. Elle peut étre

accent apporte une différenciation sémantique de deux mots, et cela selon le principe contrastif de déplacement sur l'axe horizontal de la chaîne parlée. La terminologie intonologique qualifie comme distinctives les formes intonatives (ou leurs parties) qui font diverger les modalités des phrases ou de leur parties constituantes. Une forme de conclusion intonative distinctive peut avoir la capacité de se substituer à une autre forme du même ordre de façon à modifier la modalité d'une même (partie de) la chaîne parlée. 
montante (interrogative) ou descendante (déclarative, impérative, exclamative). L'intonation interrogative est la seule marquée, parce que la différence entre les valeurs de F0 initiale et finale est, parmi les autres modalité, la seule à être négative. Sous un autre angle, l'intonation déclarative peut être considérée comme la seule réellement non-marquée, car le registre des intonations impérative et exclamative est sensiblement étendu à cause de la valeur expressive de leur réalisations.

En français, la distinctivité de l'intonation déclarative ou interrogative n'est reconnaissable que sur le dernier tiers du contour global. La différenciation de ces deux prototypes ne se fait donc en principe qu'à partir du «focus» qui est abaissé juste avant la montée finale de l'interrogation et à peine perceptiblement élevé au début de la descente vers la conclusion déclarative. Les conclusions distinctives de ces deux formes intonatives globales sont relativement longues en français: cette durée augmentée est sans doute une conséquence de fusion constante de l'événement intonatif avec l'accent d'intensité.

Les propriétés du corps intonatif ne sont pas distinctives, mais elles sont caractérisées par un trait qui semble bien contribuer à la formation du prototype global de l'intonation au niveau de la phrase. L'analyse instrumentale des phrases oralisées dans un corpus sélectionné a présenté une première proéminence locale ou globale de F0 sur la deuxième ou troisième syllabe, plus rarement sur la première, et cela sans égard à la longueur, modalité ou construction syntaxique de la phrase. L'explication de ce phénomène réside vraisemblablement dans les contraintes physiologiques du conduit vocal et dans les mécanismes psychologiques qui régissent la production de la parole. L'émanation «précoce» du F0 doit faire partie de la stratégie fondamentale de la parole, à l'aide de laquelle le locuteur négocie et adresse son message parlé; elle doit relever du principe même de l'économie linguistique au niveau de la phrase. Cette propriété de la configuration du corps intonatif semble être une caractéristique générale de l'intonation phrastique pour les langues à intonation (donc, entre autres, le français), où les oppositions tonémiques n'existent pas ou ne peuvent pas pleinement remplir leur fonction linguistique.

La conclusion distinctive d'une forme intonative globale ou locale se réalise par une variation de la hauteur tonale dont le rendement est maximal très exactement sur la dernière syllabe de l'unité intonative ${ }^{7}$ - et donc aussi de l'unité accentuelle. En conséquence principale de ce fait, la partie distinctive de l'intonation recouvre la position extrêmement prédictible de l'accent syntagmatique fixe qui subit sans doute une sorte d'effacement à l'oreille d'un locuteur français. À savoir, celui-ci n'est capable de percevoir ni la réalisation de l'accent d'intensité en cette position finale précise ni de rendre compte pratiquement de ses fonctions linguistiques. Tout cela signifie que les

7 Ces conclusions intonatives locales sont en rapport de cohérence non seulement avec l'unité accentuelle, mais aussi avec la structure syntaxique de la phrase. Leur réalisations tonales se résument en principe en trois types de conclusions intonatives: progrédience (continuation), terminalité (finalité) et suspension (incidence). 
réalisations intonatives françaises doivent être caractérisées par une variation tonale relativement radicale; le registre dans lequel elles se situent est en général plus ample que les registres relatifs dans lesquels opèrent les formes intonatives des langues à accent mobile.

\section{Intensité accentuelle et variation intonative: rendez-vous de deux proéminences prosodiques}

Cette étude étant concentrée sur la prosodie de la syllabe finale d'une unité accentuelle ou intonative, il est sans doute incontournable d'entâmer un examen bref et concis du côté physique de sa réalisation, autrement dit, d'examiner la substance de ce fragment de la chaîne parlée. Tout élément de réalisation parlée est mesurable acoustiquement au moyen de trois paramètres qui tour à tour participent à la formation d'une manifestation prosodique quelconque et à la constitution de sa forme par laquelle doivent être reconnaissables ses fonctions. «Nous supposons que l'accent comme démarcateur, dans ses différentes fonctions peut être défini seulement au cours d'une recherche effectuée dans le cadre d'un texte oral prononcé et reçu, dont la segmentation est conditionnée avant tout par l'intonation du locuteur, (souligné par P.V.) par la tendance communicative du texte en relation avec l'ensemble des conditions dans lesquelles a lieu le discours, et avec le destinataire de ce discours. ${ }^{8}$

La syllabe accentuée en français présente donc un cas extrêmement intéressant pour deux raisons. Premièrement, sa position entraîne une situation de «double proéminence» syllabique, c'est-à-dire une valorisation prépondérante, simultanément par l'énergie expiratoire et par la variation tonale. Touchée donc par une fusion constante de deux activités prosodiques qui se partagent le même champ d'action, la syllabe finale du syntagme français porte l'accent qui, d'habitude appelé «accent d'intensité», devient en réalité un accent de phrase. Deuxièmement, le troisième paramètre prosodique, celui de durée vocalique, se joint nécessairement à cette réalisation «tonique» de la syllabe proéminente en position finale. La réalisation physique d'une telle syllabe dans la dimension temporelle présuppose ainsi une valeur également proéminente de la durée. Même si l'on rappelle que chaque voyelle (et il est en fait question des voyelles figurant en tant qu'éléments nucléaires des syllabes) possède des particularités temporelles inhérentes et qu'en conséquence de ce fait une syllabe intrinsèquement courte pourrait, en position accentuée, ne pas être la plus longue de l'unité accentuelle, il n'est pas moins justifié de constater avec assurance le fait suivant: la voyelle qui, en français, forme le noyau d'une syllabe en position d'accent de phrase se distingue régulièrement des autres voyelles d'une même unité accentuelle par une valeur relativement supérieure de durée. Cette augmentation de valeur temporelle est donc la conséquence de cette double action de l'accent et de l'intonation sur une seule syllabe. 


\section{Une étape synchronique dans l'évolution du système accentuel français}

La réflexion à propos de ce statut indubitablement particulier de la syllabe accentuée en français peut être menée dans au moins trois directions, chacune d'elles proposant une solution, ou du moins une tentative d'expliquer le phénomène d'une manière autonome. La première tend à suggérer que l'accent d'intensité est en voie de perdre sa fonction démarcative, la deuxième essaie de démontrer l'affaiblissement systématique de la fixité de l'accent français, et la troisième se propose à résumer en quelque sorte les deux premières en présumant un changement radical dans la nature même du fait prosodique, connu sous le nom d'accent d'intensité, et en admettant à la fois la préservation de cet accent, mais dans un rôle différent.

\section{1) Français, langue sans accent}

«En raison du syncrétisme de l'accent et de l'intonation dans une unité, le syntagme, où seule la modulation - au sens glossématicien du terme - assume une fonction linguistique, on ne peut pas identifier l'accent comme une unité indépendante. ${ }^{9}$ Mario Rossi, intonologue aixois, reprend en quelque sorte ici l'opinion de Knud Togeby ${ }^{10}$ qui tente d'avancer que le français est une langue sans accent. Puisque l'accent en français est fixe, postule Togeby, il ne se trouve pas en position d'assumer les fonctions morphologiques ou sémantiques; il est, en plus, dépourvu de faculté distinctive au sens phonologique du terme. Mais Rossi ne s'arrête pas sur la commodité spéculative de ces constatations. Il problématise la chose en mettant en doute les deux méthodes habituelles de traiter les rapports entre la substance et la forme de l'accent en français: d'un côté l'analyse expérimentale de la nature de ce fait prosodique qui ne saurait pas, de manière satisfaisante, rendre compte de ses fonctions, et de l'autre la méthode formelle et fonctionnelle qu'est l'analyse auditive, à travers laquelle les auditeurs ne sont pas capables de dire avec assurance si une mise en relief perçue est due à une augmentation de l'intensité ou de la durée ou encore à une variation de la hauteur tonale. A travers l'exécution d'une étude du problème, considéré selon une méthodologie hypothético-déductive, développée et établie par le grand phonéticien suédois Malmberg, ${ }^{11}$ Rossi est amené à conclure que «le français est une langue sans accent, en ce sens que l'accent et l'intonation ne constituent, ni par leur nature, ni par leur fonction, deux unités distinctes. Mais il reste que les morphèmes et les lexèmes ont des propriétés accentuelles.» ${ }^{12}$ Dans cette orientation intonologique, issue de la théorie transformationniste, une forme accentuelle (accentème) doit figurer dans la structure profonde du morphème concerné pour pouvoir ensuite générer l'intonation sur le même élément de la châne parlée. Le résultat d'une telle opération prend une forme intonative distinctive que les intonologues appellent intonème.

10 Togeby, K. (1965), pp. 31, 32, 37 et 53.

11 Malmberg, B. (1969), pp. 220-242.

12 Rossi, M. (1979), p. 39. 


\section{2) Mobilisation de l'accent en français}

Un autre traitement possible des conséquences de la fusion de l'intensité et de la variation intonative sur la dernière syllabe du syntagme français, est représenté par l'observation d'une évolution du déplacement plus ou moins systématique de l'accent vers la gauche sur 1'axe horizontale de la chaîne parlée ou, en termes temporels, une certaine anticipation de l'accent. Cette explication, conçue par le phonéticien hongrois, Ivan Fónagy, pose des questions sérieuses sur la fixité de l'accent français sans parler cependant de ce qui, d'après la terminologie courante, est appelé «accent d'insistance». «L'accent français nous offre, à n'en point douter, le rare spectacle d'un système prosodique en évolution. (...) Ce changement prosodique en cours auquel nous avons la chance d'assister et de participer (...) ne se fait pas en même temps chez tous les locuteurs, et dans l'ensemble du vocabuiaire; il n'est pas indépendant de la signification des mots, il n'est pas mécanique, et les modifications ne sont pas inconscientes. (...) Ce n'est donc pas le caractère automatique de la phonation, de l'accentuation, de l'articulation sonore qui nous empêche de prendre conscience d'un changement en cours, mais, au contraire, la fonctionnalisation des phénomènes sonores déviants (souligné par P.V.) qui se trouvent presque immédiatement intégrés au système, situés dans un espace virtuel où chaque variante aura une place qui lui sera propre. ${ }^{13}$ L'interprétation de Fónagy rend compte, d'une façon explicite, d'une certaine perte tout au moins partielle du rendement fonctionnel qu'est censé d'ailleurs produire l' «accent d'intensité» affectant la dernière syllabe de l'unité accentuelle. Si l'on s'appuie, en effet, sur l'observation du discours médiatique (surtout le discours diffusé par la radio et télévision) et de quelques autres types de discours public, on aperçoit aisément, sans même être expert en la matière, une tendance persistante qu'ont les locuteurs à accentuer les syllabes qui, selon un regard normatif sur la structuration de l'unité accentuelle, seraient qualifiées inaccentuées ou même inaccentuables (diverses clitiques, par exemple). C'est certainement une manière de parler qui peut refléter une attitude ou le désir de démontrer une qualité professionnelle, mais c'est aussi certainement autre chose que simple utilisation des «accents d'insistance» qui visent le plus souvent à traduire des expressions émotives. Ce déplacement de la position de l'accent d'intensité, devenu en un sens mobile, est sans doute la conséquence d'un affaiblissement sinon d'une perte apparente de fonction démarcative que la variation intonative exerce, à la place de l'accent, sur la syllabe finale du syntagme.

\section{3) Retour aux origines d'un accent tonique}

Une troisième interprétation des résultats de la double activité prosodique sur la syllabe fonctionnellement accentuée en français essaie de résumer les positions qu'ont prises Rossi et Fónagy à ce propos, en indiquant en même temps qu'un type nouveau d'accent syntagmatique est en train de se constituer en français moderne. Il s'agirait bien d'un accent qui reste en position de préserver sa fonction essentielle, donc 
fonction démarcative, mais dont la substance réalisatrice n'est pas la même. Il semble en effet que cet accent ne réalise plus sa fonction au moyen d'une augmentation quantitative de l'énergie expiratoire. Sous l'action de toute la variété des conclusions intonatives possibles, la nature matérielle de ce fait prosodique se trouve essentiellement modifiée. Là où l'intensité vocalique était censée délimiter les unités accentuelles, c'est réellement la variation de la hauteur tonale qui, maintenant, semble opérer simultanément sur la réalisation de deux faits prosodiques. C'est une sorte de «co-opération» qui, en termes de dépendance ou de coïncidence entre divers systèmes linguistiques (prosodiques), pourrait être qualifiée comme redondante. Cependant, un autre point de vue sur la question s'impose en proposant une solution plus productible. Si l'on considère la production d'un message parlé sous l'angle de sa fonction communicative, il est justifié de voir dans la combinaison des actions de divers systèmes linguistiques un principe de cohérence qui se trouve au fondement même de la structuration convenable d'une chaîne parlée. Selon cette manière de voir les choses, un rendez-vous de deux structures prosodiques (accent et intonation) ne sera pas "accusé» de redondance; on trouvera de la raison à interpréter les propriétés de ce point d'encrage comme un fonctionnement de deux degrés de liberté différents à l'intérieur d'un même système complexe, à savoir celui dont les divers systèmes (entre autres accent et intonation) contribuent, à travers une co-opération, à la construction d'un message parlé cohérent et intentionné, acceptable à la perception, à l'analyse et à la compréhension, procédés physiologiques et psychologiques par lesquels un interlocuteur est capable d'accepter un message en tant que message bien fait.

A travers l'action simultanée et substantiellement prépondérante de l'intonation, l'accent de phrase français est donc en train de se transformer, toujours du point de vue de sa nature, en un type d'accent tonique,$^{14}$ sa fonction demeurant en rapport étroit de cohérence avec la distinctivité intonative qui détermine la modalité d'une unité accentuelle ou d'une phrase toute entière. Ce point appelle à une brève explication de cette désignation renouvelée de l'accent démarcatif.

Chez les grammairiens de l'Antiquité, le terme d'accent tonique était réservé à l'accent de hauteur ou ton, seul connu en grec ancien et en latin classique. Il a fini par désigner, en linguistique formaliste de ces deux derniers siècles, l'accent de force ou, plus communément encore, accent d'intensité, propre aux systèmes accentuels des langues à accent fixe comme à ceux à accent non-prévisible. Ce glissement de sens témoigne d'un manque de clarté terminologique à propos de la diversification des faits prosodiques. Il n'est, pour le moins, pas aisé de saisir de prime abord que «accent d'intensité» et «accent tonique» sont censés désigner la même chose dans le système prosodique du français.

14 Encore faut-il souligner que l'accent tonique doit être nettement distingué de l'accent appelé tonématique qui, lui, désigne un fait prosodique fonctionnant selon le principe phonologique de substitution et appartient aux phonétismes des langues à ton. 
Dans l'optique de la présente réflexion, l'accent tonique donne donc une dénomination à l'accent syntagmatique du français qui se révèle ne pas être réalisé par l'action d'une intensité relativement accrue, mais par une variation de la hauteur tonale, provenant de la cohérence avec la réalisation de la conclusion intonative sur la même syllabe. En co-opération avec le fonctionnement des intonèmes qui modalisent la partie concernée de la chaîne parlée, il semble donc que cet accent préserve sa fonction démarcative.

Cependant il apparaît que la réalisation de véritables accents d'intensité persiste en affectant d'autres syllabes d'une unité accentuelle, souvent celles qui, dans les schémas accentuels «didactiques», sont généralement reconnues comme non accentogènes. Il s'agit d'abord de positions accentuelles dont la réalisation peut servir à exprimer une insistance émotive ou logique. En plus, l'intensité semble également opérer dans l'accentuation de syllabes traitées normalement comme inaccentuables, mais qui dans la diversité des discours médiatiques reçoivent de plus en plus systématiquement la marque de l'accent et font en quelque sorte «refonctionner» l'accent d'intensité qui, en français, a évidemment fini par perdre son pouvoir à la dernière syllabe du syntagme.

\section{BIBLIOGRAPHIE}

BARIŠNIKOVA, K. K. (1979): À propos des accents de phrase et de la proéminence dans la langue française contemporaine, in: Studia Phonetica 17, Didier, Ottawa, pp. 103-109.

BOLINGER, Dwight L. (1955): Intersections of Stress and Intonation, in: Word 11 (2), pp. 195-203.

FÓNAGY, Ivan (1979): L'accent français: accent probabilitaire. Dynamique d'un changement prosodique, in: L'accent en français contemporain, Studia Phonetica 15, Didier, Ottawa, pp. 123-233.

GARDE, Paul (1968): L'accent, Presses Universitaires Françaises, Paris.

MALMBERG, Bertil (1969): Phonétique française, Hermods, Malmö.

ROSSI, Mario (1979): Le français, langue sans accent?, in: L'accent en français contemporain, Studia Phonetica 15, Didier, Ottawa, pp. 13-51.

TOGEBY, Knud (1965): Structure imanente de la langue française, Larousse, Paris.

VITEZ, Primož, AUBERGÉ, Véronique (1995): The Intonation Gesture of Slovene: first indications, v: Proceedings of 4th European Conference on Speech Communication and Technology, Madrid.

VITEZ, Primož (1995): Analyse contrastive de l'intonation phrastique en français et en slovène, v: Linguistica XXXV, 2, str. 257-274, Ljubljana.

\section{Povzetek}

JAKOSTNI NAGLAS IN DELOVANJE STAVČNE INTONACIJE V SODOBNI FRANCOŠČINI

Avtor predstavlja nekatera poglavitna teoretska in praktična izhodišča razmisleka o načinih, kako lahko delovanje stavčne intonacije $v$ francoščini vpliva na značilnosti jakostnega naglasa, na njegovo naravo in njegove funkcije, zlasti razmejitveno. 
Po splošno uveljavljenem mnenju je jakostni naglas v francoščini prozodično sredstvo, ki v okviru sintagme kot naglasne enote označuje zadnji zlog. Prav zadnji zlog v sintagmi pa je hkrati tudi tisti del govorne verige, na katerem se v francoščini najbolj razvidno uresničujejo intonacijski zaključki. Gre torej za pojav, značilen za francoski prozodični sistem, pri katerem en sam zlog odločilno zaznamujeta razločevalni vrednosti dveh glasovnih parametrov, namreč jakosti in tonske višine, tema dvema pa je redno dodan še tretji, namreč izrazitejše trajanje jedrnega samoglasnika.

Razmislek o posledicah te dvojne oznamovanosti zadnjega zloga v francoski naglasni in intonacijski enoti pelje $\mathrm{k}$ vsaj trem vrstam možnih sklepov. Prvi je ta, da je francoščina v določenem smislu jezik brez naglasa, kar pomeni, da je razmejitveno funkcijo naglasa v celoti prevzela intonacija. Druga razlaga sklepa, da izguba funkcionalnosti francoskega jakostnega naglasa na zadnjem zlogu sintagme povzroča sistematično premikanje naglasa $v$ levo, in sicer v skladu $z$ govorčevo zavestjo o namenu govornega sporočila. Po tej razlagi v francoščini poteka korenita sprememba naglasnega sistema, vsled katere jakostni naglas dobiva novo funkcijo $\mathrm{v}$ oblikovanju francoske govorne verige. Tretja interpretacija skuša povzeti obe prejšnji in nakazuje, da se v francoščini tvori nov tip sintagmatskega naglasa, ki ohranja svojo razmejitveno funkcijo na zadnjem zlogu, vendar pa ima po mnenju avtorja drugačno snovno podobo. Razmejitveni naglas pod vplivom delovanja intonacije namreč zadobiva značilnosti toničnega naglasa, njegova jezikovna vloga pa je $\mathrm{v}$ funkcionalni sovisnosti $\mathrm{z}$ razločevalno vrednostjo intonemov, ki posameznim naglasnim enotam določajo intonacijsko modalnost. Hkrati se zdi, da se jakostni naglas ohranja, vendar v naglasni enoti zavzema druge položaje, pogosto tudi na tistih zlogih, za katere v splošnem velja, da so lahko zaznamovani s čustvenimi ali logičnimi poudarki. 